\title{
El comportamiento residencial de los mayores. Análisis biográfico de la movilidad en la vejez
}

Dolores Puga Instituto de Economía y Geografía, CSIC dpuga@ieg.csic.es

RESUMEN

En las actuales sociedades europeas envejecidas, los comportamientos residenciales de las personas de edad están adquiriendo una importancia creciente. En este artículo se exploran las relaciones causales entre la biografía migratoria previa y las pautas residenciales adoptadas tras los 55 años, usando Event History Analysis. Los resultados demuestran que el lugar de nacimiento marca la trayectoria migratoria posterior de los individuos, llegando a afectar incluso al comportamiento residencial en la vejez. La existencia de migraciones previas, el hábitat de residencia, así como la duración del período sedentario, ejercen también una importante influencia sobre la decisión de movilidad en la vejez. Finalmente, se demuestra que la reducción de la movilidad entre las generaciones de mayores más recientes es un efecto de su biografía migratoria previa. Las trayectorias migratorias protagonizadas por los individuos a lo largo de su vida, joven y adulta, se revelan más decisivas respecto a los comportamientos residenciales en la vejez que las circunstancias coyunturales. Por ello, posibles cambios en las biografías migratorias de las generaciones más jóvenes podrían traducirse en cambios en los niveles de movilidad en la vejez, independientemente de las circunstancias coyunturales. A este respecto se pueden observar diferencias por género, mostrándose las mujeres más vulnerables frente a las circunstancias históricas que los hombres.

Palabras clave: Envejecimiento, Estrategias Residenciales, Movilidad, Calidad de Vida en la Vejez, Curso de Vida, Biografía Migratoria, Análisis Biográfico. 


\section{INTRODUCCIÓN}

La movilidad es un proceso esencial en la evolución de la población y refleja fenómenos sociales muy significativos. Uno de los componentes más importantes de la misma, y de presencia creciente, son los movimientos residenciales de los mayores. Como consecuencia no sólo de una sociedad más envejecida, sino también de una sociedad que brinda nuevas opciones y oportunidades a las personas de edad, comienzan a extenderse a estas edades fenómenos demográficos hasta la actualidad más presentes en otros momentos del curso de vida, entre ellos la movilidad residencial. El conocimiento de la movilidad durante el curso de vida avanzado nos ayudará a entender mejor los cambios que se producirán en nuestra sociedad envejecida, en el futuro inmediato. En este artículo se plantea un acercamiento a los comportamientos abordados en la vejez, y en especial a los mecanismos subyacentes tras los mismos.

La vejez se traduce, en muchos casos, en una menor interacción con el contexto social. Por ello, los comportamientos adoptados durante la misma están menos condicionados por características exógenas, y más por la ya larga trayectoria vital del individuo. A edades avanzadas, el individuo está menos condicionado por el presente o por el futuro -mercado laboral, lugar de trabajo, formación familiar o aumento de la familia- que por el pasado: lugar en el que se ha nacido y donde se encuentran las redes familiares, lugares en donde se ha vivido, lugares en los que se han establecido los hijos, situación económica alcanzada, preferencias, aficiones y actitudes que se han ido afianzando; es decir, todo un sistema de preferencias, posibilidades y restricciones, herencia de nuestra propia trayectoria biográfica. Así pues, para entender el porqué y el cómo de las decisiones residenciales de los mayores ha sido necesario adentrarse en su historia vital. De esta forma, el curso de vida ha proporcionado una inestimable lista de razones, motivos y aspiraciones para entender los comportamientos residenciales en la vejez.

\section{MARCO CONCEPTUAL}

Los movimientos residenciales de las personas mayores han sido objeto de poca atención por parte de sociólogos, demógrafos, economistas y geógrafos, debido en parte al escaso número de personas y hogares implicados en estos movimientos residenciales. Pero también porque la investigación sobre la migración se ha centrado casi exclusivamente en la redistribución de la fuerza de trabajo y sus consecuencias, con la consecuente endeblez teórica de estos movimientos, para cuyo estudio se han utilizado conceptos y principios de las migraciones económicas (Flynn et al., 1985). La movilidad residencial de los mayores no sólo es relevante por la novedad de la adopción de estrategias de movilidad en la vejez, 
sino también por el importante papel que los mayores pueden desempeñar en el mercado inmobiliario. Por una parte, las personas de edad demandan un determinado tipo de viviendas; por otra, pueden ocupar, o dejar disponibles, casas atractivas para otro tipo de hogares. En cierta medida, la movilidad de hogares jóvenes hacia viviendas en centros urbanos está frenada, a su vez, por las imposibilidades de los mayores de moverse de ellas.

En la mayoría de las sociedades occidentales los jóvenes muestran unas probabilidades de migración mucho más altas que las de los mayores (Law y Warnes, 1982; Vergoossen y Warnes, 1989; Rogers y Castro, 1984). Tras estos planteamientos, muchas de las conclusiones obtenidas eran que las personas de edad se encontraban satisfechas con su situación residencial. Sin embargo, algunos estudios han demostrado que muchos mayores se quedan en áreas urbanas deterioradas que les resultan poco satisfactorias hasta mucho después del momento en que les hubiese gustado marcharse (Varady, 1980). En realidad, las personas mayores han tenido mucha menos facilidad para realizar sus deseos y planes de movilidad que los jóvenes.

En la actualidad se observan unas condiciones — unas edades de jubilación relativamente tempranas, mejoras en salud y calidad de vida, así como sistemas de soporte económico para la jubilación - que se han traducido en una vejez más larga, y en un incremento del número de personas que tienen los medios suficientes para adoptar una estrategia residencial de movilidad en las últimas etapas de su curso de vida, añadiendo la migración al abanico de opciones residenciales postjubilatorias (Cribier, 1980). La vejez ha pasado de ser un corto período asociado a la debilidad, para convertirse en una tercera edad de la vida durante la cual se proponen nuevas actividades de ocio y sociales (Laslett, 1991). Pero tras este primer período saludable ganado a la morbilidad y a la mortalidad empiezan a aparecer problemas de discapacidad y dependencia, que dificultan paulatinamente el mantenimiento de la autonomía personal y la independencia residencial, lo que se puede traducir en una nueva estrategia de movilidad.

Dado que la movilidad en la vejez presenta unos patrones particulares, las explicaciones relacionadas con el mercado laboral no son satisfactorias para explicar este tipo de movilidad. Gran cantidad de estudios sobre la movilidad en la vejez parten de la perspectiva del proceso de toma de decisión de la migración. Se dispone en la actualidad, por tanto, de diversos modelos que no difieren en gran medida unos de otros, pero que en conjunto han contribuido a completar los conceptos que han ido incrementando nuestro conocimiento del proceso de movilidad y de la toma de decisión de la misma (Cribier, 1975, 1994; Wiseman, 1980; Serow, 1987; Clark y Wolf, 1992; Cribier y Kych, 1992, 1993; Abellán, 1993). El proceso de decisión concerniente a la elección de una situación residencial se ha de considerar como parte de una estrategia global de vida de las personas mayores. Ésta está 
guiada por sus preferencias, deseos, valores y normas personales. Pero también se ha de tener en cuenta que la persona mayor no está aislada, sino que está inmersa en un conjunto de factores, normas, valores y actitudes predominantes en el contexto social en el que vive (país, región, comunidad o red social).

Estos modelos son empleados habitualmente para explicar el proceso migratorio de los mayores, pero algunos estudios han demostrado que la movilidad adoptada en la vejez en relación con situaciones similares - como la relacionada con los cambios en las necesidades de la vivienda o en el entorno, o el deterioro del estado de salud- no es seguida por una secuencia migratoria homogénea ni universal (Manton, 1988; Worobey y Angel, 1990; Angel et al., 1992). Las investigaciones sobre los procesos de decisión migratoria en las últimas etapas de la vida deben llevarnos a modelos teóricos en los que figuren tanto las motivaciones de los individuos como los factores estructurales y biográficos que determinan sus elecciones. Las posibilidades de realizar las propias preferencias, y de conseguir los resultados perseguidos mediante una estrategia residencial determinada, están fuertemente condicionadas por un conjunto de oportunidades y de restricciones. Entre ellas hay que mencionar las provenientes de la propia trayectoria vital del individuo.

Cuando estudiamos la migración habitualmente olvidamos lo que ésta tiene de ruptura, de redefinición de actividades cotidianas, de modificación de espacios, de relaciones, de referencias, conceptualizándola como producto de variables económicas o provenientes del mercado inmobiliario. Incluso en los casos en los que la movilidad aparece íntimamente ligada a significativos eventos del curso de vida como la jubilación, a la hora de explicar las decisiones residenciales a menudo aludimos a las dificultades materiales o a la adecuación de la vivienda (Wiseman, 1980; Stokols y Shumaker, 1982; Meyer y Speare, 1985). Un cambio de residencia es un evento en torno al que se construye o reconstruye la biografía de un individuo (Warnes, 1993). Supone un nuevo inicio, pero al mismo tiempo también significa una ruptura con un espacio de vida y unas redes de relaciones previas. Se traduce no solamente en un cambio geográfico, sino también en un cambio más o menos profundo de las actividades diarias, de las prácticas sociales; altera las fidelidades, valores y ataduras de los individuos respecto a su espacio físico y social habitual.

Pero esta ruptura, esta profunda modificación asociada al cambio espacial, no constituye habitualmente un fin en sí mismo, sino que se trata de un medio para obtener una mejora determinada o para ajustarse a una nueva situación, un comportamiento instrumental (Willekens, 1991). En el curso de vida, los comportamientos migratorios están subordinados a las posibilidades y restricciones aportadas por las diferentes trayectorias biográficas del individuo (de hogar y familia, educación y ocupación, y migratoria y de vivienda previa), entendiendo, por tanto, las estrategias residenciales como un mecanismo de ajuste en un 
momento determinado. El curso de vida proporciona, por tanto, una inestimable lista de razones, motivos y aspiraciones para entender y explicar las estrategias residenciales en la vejez (Meyer, 1986). En este artículo se adopta, por lo tanto, una perspectiva longitudinal, entendiendo la biografía individual como variable significativa para comprender e interpretar las elecciones residenciales.

Un factor explicativo de la elección residencial en la vejez será la biografía individual previa. Para ello partimos del concepto de continuidad biográfica, por el cual las acciones pasadas de los individuos condicionan los medios y capacidades que han acumulado y, por lo tanto, condicionan sus acciones futuras (Elchardus, 1984). En realidad, conducimos nuestras vidas a través de trayectorias razonablemente consistentes que llamamos biografías (Mulder, 1993). Las elecciones hechas en cualquiera de estas biografías dibujan con mayor o menor precisión una continuidad biográfica individual, por lo que las elecciones residenciales en la vejez se ven parcialmente determinadas por la biografía previa del individuo. Por ello, se estudia en este artículo la relación entre la movilidad en la vejez y la historia migratoria previa del individuo.

\section{FUENTE}

Las fuentes habituales para el estudio de movimientos migratorios, como los Censos de Población o las Estadísticas de Variaciones Residenciales (EVR) del Instituto Nacional de Estadística, tienen la bondad de permitir obtener series temporales de los mismos, lo que posibilita el estudio de la evolución del fenómeno. Al mismo tiempo, debido a que se trata de registros exhaustivos, permiten un mayor detalle territorial que los datos de encuesta, siempre limitados por los problemas inherentes al tamaño de la muestra. Como contraposición, estas fuentes estadísticas clásicas tan sólo proporcionan un número muy limitado de variables, en su mayoría referentes a las características del momento censal o en el que se realiza el registro, no a las características previas al fenómeno migratorio que permitirían establecer relaciones causales. Se trata, por tanto, de fuentes que aportan información transversal, pero que no recogen información retrospectiva, lo que impide la reconstrucción y el estudio del curso de vida previo del individuo. Por otra parte, este tipo de fuentes ofrecen, generalmente, datos agregados (con la excepción, en España, del último Censo de Población, de 1991, del que está disponible una muestra de los registros individuales), lo que imposibilita la aplicación de metodologías de análisis individual que permitan establecer relaciones causa-efecto entre la trayectoria previa de los individuos y su comportamiento en la vejez.

Por estos motivos, la fuente utilizada para este estudio es la Encuesta Sociodemográfica de 1991 (ESD91), realizada por el Instituto Nacional de Estadística en el último cuatrimes- 
tre de dicho añoํ. En contraposición con las fuentes clásicas, los datos de encuesta permiten realizar un análisis individual de la información, lo que facilita la inferencia causal entre las variables explicativas y el fenómeno demográfico estudiado y permite evaluar su efecto.

La ESD91 aporta una gran cantidad de información demográfica retrospectiva, con un buen nivel de detalle temporal, fundamental a la hora de aplicar técnicas de análisis biográfico. La información recogida en el amplio cuestionario de la encuesta permite reconstruir la biografía migratoria, laboral y familiar de cada individuo (a partir de los eventos biográficos reportados). Por otra parte, el carácter retrospectivo de la encuesta permite poner en relación un evento con las características del sujeto en el momento que se produjo, y no en la actualidad, que puede representar más una consecuencia que una causa del mismo.

A la gran cantidad de información aportada, su carácter retrospectivo, así como la posibilidad de análisis individual, hay que añadir otro de los principales atractivos de la fuente, que es su importante tamaño muestral: para individuos mayores de 55 años (entre 55 y 89 años) contamos con una muestra de 57.955 sujetos (24.473 hombres y 33.482 mujeres), muestra nacional suficientemente amplia como para permitir un amplio nivel de desagregación y detalle en el análisis.

Pero en este tipo de fuentes, obviamente, no todo son bondades. Aunque los problemas habituales del trabajo con encuestas, derivados de las limitaciones debidas al tamaño de la muestra, no suponen excesivos problemas en este caso, dada la amplitud de la misma, nos enfrentamos a otros problemas derivados de su carácter retrospectivo.

En primer lugar, se ha de contemplar el riesgo de posibles fallos de memoria respecto a los eventos reportados por los individuos, especialmente teniendo en cuenta que se trata de población mayor a la que en muchos casos se le pregunta sobre sucesos que tuvieron lugar hace varias décadas. Asumiendo las posibles inexactitudes en la declaración, un desplazamiento migratorio es un evento lo suficientemente importante en una vida como para no olvidarlo fácilmente (Courgeau, 1980), especialmente en las sociedades mediterráneas, en las que la movilidad es un fenómeno menos extendido que en las anglosajonas, por lo que, para la mayor parte de la población, una migración ha sido un evento único en su vida. Por añadidura, cotejando los datos referentes a las migraciones en su juventud con los datos históricos de registros de migraciones en el primer tercio de siglo, los resultados muestran una gran afinidad (Puga y Abellán, 1998; Nadal, 1984; García Barbancho, 1967).

\footnotetext{
1 Queremos hacer constar nuestro agradecimiento al Centre d'Estudis Demogràfics de Barcelona, y en concreto a Marc Ajen-
} jo, por la desinteresada cesión del complejo programa de control para la lectura de las variables de la encuesta. 
Finalmente, se ha de hacer referencia también a los problemas derivados de la propia naturaleza retrospectiva de la fuente. Al tratarse de una reconstrucción de las trayectorias, no contamos con los efectivos iniciales de las cohortes, sino con los supervivientes de las mismas. La diferencia entre ambos efectivos no es muy cuantiosa en el caso de las cohortes más jóvenes, pero sí se ha de tener en cuenta cuando se trata de las de edades más avanzadas. Los efectivos iniciales de estas cohortes se ven infravalorados por el efecto de la emigración internacional no retornada y el de la mortalidad. En el primero de los casos, el efecto de la emigración internacional no retornada - especialmente la que tuvo como destino América, que presenta una menor tasa de retorno- puede producir un sesgo territorial, ya que ésta fue muy fuerte desde algunas regiones de España, pero tiene una escasa incidencia a nivel nacional. En cuanto al segundo de los efectos, el de la mortalidad, se asume que este fenómeno no ha tenido una incidencia diferencial entre migrantes y sedentarios, por lo que su efecto no altera los resultados: reduce el colectivo pero no sesga la muestra, al menos en lo que al fenómeno migratorio se refiere.

\section{METODOLOGÍA}

El fenómeno demográfico objeto de estudio son los movimientos migratorios realizados con posterioridad a los 55 años, es decir, en una etapa ya de inactividad laboral o en previsión y proximidad a la misma. Se entiende por movimiento migratorio, a efectos de este estudio, cualquier cambio de domicilio que comporte un cambio de municipio de residencia, y se hará referencia a él con el término de migración o movilidad indistintamente.

El evento o transición biográfica objeto de estudio, la «migración», es susceptible de ocurrir en diversas ocasiones a lo largo de la historia de vida de un individuo. Dado que los métodos de análisis utilizados son adecuados para el estudio de eventos no renovables, es decir, que tan sólo pueden ocurrir en una ocasión a un individuo, se ha convertido el fenómeno migratorio en no renovable mediante la incorporación del rango de la migración. Es decir, a pesar de que un individuo puede cambiar de municipio de residencia repetidas veces a lo largo de su vida, tan sólo lo puede hacer por primera vez en una ocasión, al igual que por segunda vez en una sola ocasión, y así sucesivamente. Por lo tanto, una primera migración (o una migración de cualquier rango) es un suceso único en una biografía y, por consiguiente, no renovable. En este artículo el objeto de estudio será la primera migración realizada tras cumplir los 55 años (independientemente de las que se hayan podido realizar con anterioridad a esta edad).

El objetivo del estudio es evaluar el efecto que sobre migración en la vejez pueden tener diversas variables explicativas; para ello sería necesario subdividir la muestra en tantos sub- 
grupos como cruzamientos se puedan obtener entre las categorías de las variables independientes, realizando el análisis por separado para cada uno de estos subgrupos. Dicha metodología no sólo presentaría problemas a la hora de sistematizar los resultados, sino que, fundamentalmente, presenta un serio problema muestral. Incluso una muestra amplia, como es la de la ESD91, no permitiría llegar a tal fragmentación de la misma.

El método utilizado para el análisis causal de la migración será, por tanto, el análisis biográfico (Event History Analysis) con el objetivo de poder establecer relaciones causales entre variables pertenecientes a la historia migratoria previa del individuo y el comportamiento migratorio en la vejez. A partir de la observación de una serie de variables independientes, pertenecientes a la historia migratoria previa del individuo, se estudiará su efecto sobre el evento de migrar por primera vez tras los 55 años, o, lo que es lo mismo, sobre la transición biográfica que supone el paso de la situación sedentaria a la movilidad por primera vez tras los 55 años.

Para ello se ha utilizado el modelo de riesgos proporcionales de Cox, o regresión de Cox. El modelo calcula la «tasa de riesgo» (de ocurrencia del evento) o tasa de transición en función de las variables explicativas observadas, pero esta tasa toma un período de tiempo tan breve que la hace equivalente a una probabilidad (tasa instantánea). La regresión de Cox estima, por tanto, la probabilidad de transición de un estado a otro (sedentarismo/migración) en función: del tiempo individual (duración de la exposición al riesgo), del tiempo histórico (pertenencia a una cohorte) y de las características personales (variables independientes) (Teachman, 1982; Castro, 1992). De esta forma obtenemos una serie de riesgos o probabilidades relativos de ocurrencia del evento, que en cada variable están definidos respecto a una categoría o grupo de referencia. Una de las grandes bondades que presenta este método de análisis es que estima el efecto neto que tiene cada una de las variables sobre el fenómeno estudiado, controlando las posibles interrelaciones con las restantes variables introducidas en el modelo y excluyendo los efectos de las mismas. La tasa de riesgo se define como:

$$
h(t, c)=h_{0}(t) e^{B c}
$$

En donde $h(t, c)$ es la tasa de transición para un individuo con una duración de la exposición al riesgo $t$ y un vector de variables $c ; h_{0}(t)$ es la función de riesgo subyacente (baseline hazard function) correspondiente al grupo de referencia; y $B$ es el vector de coeficientes de regresión que cuantifica el efecto de cada variable independiente $\left(e^{\beta}\right), y$ que, por lo tanto, expresa los efectos de cada una de ellas como aumentos o reducciones relativos a la categoría de referencia, o, lo que es lo mismo, se obtienen riesgos relativos. 
Los resultados de este método expresan las diferencias relativas de «riesgo» de suceso del evento, que supone la pertenencia a una u otra categoría de valores para cada una de las variables contempladas. La interpretación es, por tanto, sencilla. Todos los individuos tienen un mismo riesgo subyacente que varía con la duración de la exposición al mismo (con la edad en este caso), como habremos visto mediante el análisis de tablas de vida, pero este riesgo se ve multiplicado por un determinado factor, al pertenecer a un determinado subgrupo. Este factor multiplicativo es lo que llamamos riesgo relativo, que se mantiene constante a cualquier duración, siendo el riesgo subyacente el que varía con ésta. La categoría de referencia (aparecen entre corchetes en las tablas de resultados) tiene un riesgo 1; una categoría que presente un factor superior a éste, por ejemplo 1,25, tendrá una probabilidad un $25 \%$ superior de suceso del evento que la categoría de referencia; por el contrario, un factor menor a 1 , por ejemplo 0,75 , significa que la categoría en cuestión tiene una probabilidad de riesgo un $25 \%$ inferior a la de referencia.

\section{ESTRATEGIA RESIDENCIAL EN LA VEJEZ: EL CURSO DE VIDA COMO DETERMINANTE}

De continuar las actuales pautas de movilidad en la vejez, un 15\% de los nacidos antes de 1936 habrá realizado, al finalizar su trayectoria de vida ${ }^{2}$, al menos, un movimiento migratorio con posterioridad a los 55 años (Puga, 2000). Este artículo pretende indagar en la causa de por qué un sexto de los individuos de estas generaciones, a idénticas edades y en similares situaciones, toman la opción de la movilidad, frente a la estrategia sedentaria del resto de la población. Se plantea para ello el estudio del papel que juega la historia migratoria previa como determinante de las opciones que un individuo tiene de migrar con posterioridad a los 55 años cumplidos.

Se han introducido en el análisis cinco variables independientes, además de la cohorte de nacimiento y el sexo; cuatro de ellas son variables resumen de la historia migratoria, y la última es la tenencia de la vivienda previa a la migración, que se ha demostrado significativa en numerosos estudios sobre el tema (Roseman, 1971; Golant, 1982; Speare y Meyer, 1988). Las variables independientes observadas, pertenecientes a la historia migratoria, son: el tamaño demográfico del lugar de nacimiento (denominado también hábitat de nacimiento), el número de migraciones previas, la duración del período sedentario o años que llevaba el individuo residiendo en el mismo municipio en el momento de la migración (de llegar ésta a producirse, o en la actualidad, en su defecto), y el tamaño demográfico del

2 El cálculo se ha obtenido a través de una tabla de vida: aplicando las probabilidades por edades, calculadas a partir de los movimientos migratorios ya realizados, al total de efectivos iniciales, incluyendo aquellas generaciones más jóvenes cuyo período de «exposición al riesgo» de migrar ha sido todavía muy reducido. 
municipio de residencia previo a la migración (o del actual en ausencia de migración), también denominado hábitat de residencia previo ${ }^{3}$.

\subsection{Análisis univariable}

En una primera aproximación, haciendo el análisis para cada una de las variables por separado (tabla 1), desde una óptica generacional, la migración en la vejez desciende entre la cohorte de edad más avanzada y la más joven. Tomando como categoría de referencia (se encuentra entre corchetes) la cohorte nacida entre 1902 y 1906, es decir, los de edades más avanzadas, se puede observar que éstos han tenido una probabilidad de migración tras los 55 años hasta tres veces mayor que los nacidos treinta años más tarde. En realidad, las generaciones nacidas durante la primera quincena del siglo han estado expuestas a unas probabilidades de migración muy similares (con diferencias máximas en torno a un 10\%), acusándose un descenso en las probabilidades de movilidad tan sólo para los nacidos con posterioridad a 1917. Como resultado de esta evolución, las probabilidades experimentadas por las generaciones nacidas entre 1927 y 1931 son ya mucho menores, y para las nacidas entre 1932 y 1936 suponen tan sólo la mitad y un tercio, respectivamente, de los niveles de probabilidad de migración a los que estaba expuesta la cohorte inicial. Los patrones por género no arrojan resultados diferentes para hombres y mujeres: la migración en la vejez es prácticamente idéntica entre ambos sexos, teniendo ellas tan sólo un riesgo un 3\% mayor.

Del análisis univariable de las principales características de la historia migratoria previa se desprende que la más significativa de todas ellas es la duración del período sedentario, o, lo que es lo mismo, el número de años que el individuo lleva residiendo en el mismo municipio en el momento de realizarse la migración. Aquellos que llevan un menor período de tiempo registran un riesgo de movilidad muy superior, que va descendiendo a mayor duración del período sedentario; así, los individuos que llevan menos de diez años residiendo en la misma área municipal tienen un riesgo de movilidad que es ya mayor del doble que aquellos que llevan cinco años más (entre diez y catorce), y que llega a ser 25 veces superior al de quienes llevan residiendo en el mismo municipio más de quince años. Este altísimo riesgo de migración afecta, por otra parte, a un porcentaje muy pequeño de esta población, ya que tan sólo un 1,6\% de los mayores de 55 años llevan menos de diez años en el mismo municipio de residencia.

\footnotetext{
3 Se han reservado para una segunda aproximación al tema variables más concretas: edad a la primera migración, patrón geográfico de la misma..., dado que ello requeriría hacer el análisis tan sólo para los individuos que han realizado algún movimiento migratorio previo a los 55 años (un 53\% del total), y en este momento se pretende abordar las estrategias residenciales del conjunto de la población mayor, incluyendo aquellos que presentan un curso de vida con ausencia de migración, dado que esta misma característica (ausencia de migración previa) puede ser relevante a la hora de plantearse la movilidad en la vejez.
} 
TABLA 1

Probabilidades relativas de migración en la vejez según historia migratoria previa

Variables independientes

Porcentaje población expuesta al riesgo

Modelo univariable Modelo multivariable

Cohorte de nacimiento

[1902-06]

1907-11

05

1912-16

07

10

1917-21

14

$1922-26$

19

1927-31

22

1932-36

23

Género

[Hombre]

45

Mujer

$1,03^{\star *}$

$0,98^{\star *}$

Duración del período sedentario

[0-9]

10-14

15 o más

96

0,99

$1,18^{\star \star}$

$0,89^{\star *}$

$1,37^{\star *}$

$0,72^{\star \star}$

$1,19^{\text {** }}$

$0,64^{\star \star}$

$1,18^{\star \star}$

$0,49^{\text {** }}$

$1,16^{\star \star}$

$0,38^{\star \star}$

$0,76^{\star \star}$

Hábitat de residencia previo

[Madrid]

Barcelona

05

$>100.000$

06

15

20.001-100.000

15

$\leq 20.000$

58

Número de migraciones previas

$[\geq 3]$

09

2

13

31

1

47

$0,62^{\star *}$

$0,43^{\star *}$

$0,04^{\star *}$

$0,42^{\text {** }}$

$0,11^{\star *}$

0

Hábitat de nacimiento

[ $\leq 5.000]$

$5.001-20.000$

38

21

$0,74^{\star *}$

$0,58^{\star \star}$

$0,79^{\star *}$

$0,34^{\star *}$

$0,52^{\star \star}$

$0,15^{\star *}$

$0,35^{\star *}$

$>20.000$

29

Madrid o Barcelona

12

$0,81^{\text {** }}$

$0,81^{\text {** }}$

$0,84^{* *}$

$0,61^{\star *}$

$0,32^{\star *}$

$0,71^{\text {** }}$

$0,44^{\star *}$

Tenencia de la vivienda

[Inquilino]

21

Propietario

30

$0,42^{* *}$

$0,77^{\text {** }}$

${ }^{* *} p<0,01$

$\mathrm{n}=57.955$

Categorías de referencia entre corchetes.

FUENTE:

Elaboración propia a partir de los datos primarios de la ESD91. 
Tras esta característica, es el hábitat (o tamaño demográfico) del municipio de residencia previo el que aporta mayores diferencias en el riesgo de migrar en la vejez. Son los individuos residentes en ámbitos metropolitanos ${ }^{4}$ los que presentan una mayor tendencia a la movilidad en estas edades, y en concreto aquellos que viven en el mayor de ellos, el área de Madrid, que presenta unas probabilidades de «expulsión» superiores en un 25\% a los de Barcelona. Los residentes en otros municipios mayores de 100.000 habitantes (capitales provinciales en su mayoría) tienen un riesgo de migración en la vejez que es tan sólo la mitad que el de los residentes en Madrid y un 25\% inferior al de los barceloneses; el de los residentes en municipios intermedios (entre 20.001 y 100.000 habitantes) es un tercio, y el de los municipios rurales (menores de 20.000 habitantes) es tan sólo un sexto del de los madrileños.

El medio urbano es percibido en muchos casos por las personas mayores como un entorno hostil: los ruidos, la contaminación, la falta de espacios adecuados para el paseo o el esparcimiento, la falta de tranquilidad... así como una percepción del mismo como un medio violento y peligroso, hacen que en muchos casos la finalización del período laboral se vea acompañada del deseo de recuperar un hábitat residencial más tranquilo y percibido como menos problemático (Wiseman, 1980; Abellán, 1993; Abellán y Puga, 1999). Pero es también en las áreas metropolitanas en donde residen la mayoría de aquellos que protagonizaron los flujos migratorios campo-ciudad — que fueron una constante a lo largo del siglo, aunque con sus momentos álgidos en los años cincuenta, sesenta y setenta-. Son estos individuos los que pueden querer recuperar el lugar de nacimiento (o de residencia previo, en el caso de haber realizado más de un movimiento migratorio a lo largo de su vida), abriendo así la posibilidad de los movimientos de retorno; son los migrantes actualmente residentes en las grandes ciudades, por decirlo de otra forma, los que tienen adonde volver. Al realizar el análisis multivariable e introducir el hábitat de nacimiento y el número de migraciones previas se podrá controlar este efecto, midiendo tan sólo el alcance que sobre el riesgo de migrar tienen las características del hábitat de residencia.

El número de migraciones previas (aquellas realizadas con anterioridad a los 55 años) se traduce en probabilidades significativamente diferentes entre los individuos que se han movido o muchas veces o ninguna. Dado que para estas cohortes la media es de un movimiento por persona, aquellos que han cambiado de municipio tres o más veces presentan una alta movilidad (siendo tan sólo el $9 \%$ de la población), mientras que los que no se han movido en ninguna ocasión son casi la mitad del colectivo (47\%). La población que ha migrado con anterioridad a esta edad en una o dos ocasiones presenta probabilidades prácti-

4 Se han utilizado como ámbitos metropolitanos las provincias de Madrid y Barcelona en su totalidad, a pesar de la no coincidencia total entre la delimitación provincial y el área urbana; ello es debido a la imposibilidad de delimitación de áreas más concretas a partir de los datos aportados por la ESD91. 
camente idénticas de migración en la vejez, siendo inferiores en un $40 \%$ a las de los más móviles, pero el doble de aquellos que presentan una historia migratoria sedentaria o con ausencia de migración. El grupo de población que ha cambiado de municipio previamente en tres o más ocasiones tiene un riesgo de migración en la vejez tres veces superior a aquellos que no han salido jamás del municipio de nacimiento. Nuevamente será el análisis multivariable el que pueda arrojar resultados más «netos» sobre el efecto de esta variable, al introducir en el mismo los hábitats tanto de nacimiento como de residencia en la vejez (previa a la migración), que pueden tener aquí un efecto enmascarado.

Entre las variables pertenecientes a la historia migratoria es el hábitat, o tamaño demográfico, del municipio de nacimiento el que parece traducirse en menores diferencias en las probabilidades relativas de migración en la vejez. La aproximación univariable demuestra que son los nacidos en municipios con un tamaño demográfico inferior a 5.000 habitantes los que tienen mayores probabilidades de migración en la vejez, un $20 \%$ superiores a los restantes individuos de sus generaciones, e incluso un $34 \%$ superiores a los nacidos en áreas metropolitanas. En realidad, esta variable es determinante de la historia migratoria posterior, dado que son los nacidos en hábitats rurales los que protagonizaron una biografía migratoria activa, realizando algún movimiento y residiendo, en gran parte, en áreas metropolitanas, o al menos urbanas, en la actualidad. Es, por tanto, necesario introducir las restantes variables de historia migratoria en el análisis para poder controlar sus efectos y así observar el efecto neto de cada una de ellas.

Respecto a la tenencia de la vivienda se demuestra que la propiedad es un fuerte vínculo con el territorio, reteniendo a la población y reduciendo las probabilidades de migrar, también en la vejez. De esta forma, los propietarios presentan unas probabilidades de migración que son menores a la mitad de las que presentan los inquilinos (un 58\% inferiores).

\subsection{Análisis multivariable}

En la aproximación multivariable (tabla 1) se observa el efecto conjunto de las variables independientes contempladas, con el objetivo de poder determinar los efectos netos de cada una de ellas sobre la migración en la vejez, eliminando los posibles efectos encubiertos de las restantes variables. Por ejemplo, la aparentemente modesta influencia del hábitat de nacimiento puede estar encubriendo los efectos de variables relacionadas con éste, como el número de migraciones realizadas con anterioridad o el hábitat de residencia previo a la migración; introduciendo conjuntamente las variables en el análisis obtenemos las probabilidades depuradas correspondientes a cada una de ellas, eliminando las posibles interrelaciones. 
En primer lugar, se puede observar la influencia que sobre la historia migratoria de los sujetos de estas generaciones ha tenido el momento de nacimiento, dado que, al introducir las variables resumen de dicha historia, el peso de la cohorte de nacimiento cambia no sólo de magnitud, sino incluso de signo para todas las generaciones, excepto para la última. Así se revela que el descenso del riesgo de migración en la vejez que sufren las generaciones nacidas entre 1907 y 1926 no es debido a un efecto generacional (o, lo que es lo mismo, a la influencia del momento histórico), sino que es efecto de la diferente trayectoria migratoria vivida por estos individuos.

El efecto generacional no sólo no restringió la migración de esta población, sino que fue muy favorable para aquellos nacidos entre 1912 y 1916, que, a idéntica biografía migratoria, muestran unas probabilidades de migración en la vejez un $37 \%$ superiores a las de la cohorte inicial. Las restantes generaciones nacidas en las tres primeras décadas del siglo presentan también unas probabilidades de migración en la vejez entre un 16 y un 19\% superiores a las de las de edades más avanzadas; sufriendo tan sólo los más jóvenes (nacidos entre 1932 y 1936) una coyuntura claramente negativa para la migración en la vejez, que se ve reducida en un cuarto respecto a la primera generación, e incluso en un $60 \%$ respecto a los nacidos entre 1912 y 1916.

Es decir, un individuo nacido durante la veintena 1907-26, en el caso de que tuviese una biografía migratoria idéntica a la de otro nacido a principios de siglo, estaría expuesto a un riesgo de migración en la vejez igual o superior al de aquél; por lo que las menores probabilidades de migración en la vejez que resultan cuando se observa tan sólo el efecto de la cohorte (análisis univariable) hacen pensar en el gran peso que, incluso en la vejez de estos individuos, tiene la trayectoria migratoria recorrida por cada uno de ellos. La migración en la vejez es más un resultado de las necesidades y las oportunidades generadas por un ya extenso curso de vida que una respuesta a una coyuntura socioeconómica concreta a la que están más ligadas las migraciones laborales durante la etapa activa. Todo ello induce a pensar que un cambio en las biografías migratorias de generaciones más jóvenes se puede traducir en un cambio sustantivo en los niveles de movilidad cuando lleguen a la vejez, más allá de la situación coyuntural de un momento dado.

Mientras las variables correspondientes a la historia migratoria demuestran tener un claro efecto sobre las pautas de migración en la vejez que presentan las diferentes cohortes, el peso relativo de cada una de ellas sobre los movimientos residenciales en estas edades se mantiene a niveles bastante similares al introducirlas en su conjunto (tabla 1).

La única excepción es el hábitat (o tamaño demográfico) del municipio de nacimiento (que en gran parte de los casos no coincide con el de residencia actual), que, una vez controla- 
das las restantes variables migratorias, muestra un claro efecto diferenciador. Son aquellos nacidos en hábitats rurales y municipios urbanos pequeños (menores de 20.000) los que presentan unas probabilidades de migración en la vejez hasta 20 y 25 veces superiores a las de los nacidos en áreas metropolitanas. Las diferencias son menores respecto a las áreas urbanas mayores de 20.000 habitantes, que muestran unas probabilidades de migración entre 8 y 10 veces superiores a las de los municipios más pequeños.

Fue el hábitat de nacimiento el que determinó en gran medida la trayectoria migratoria posterior. Los nacidos en áreas metropolitanas se podría decir que nacieron «en el lugar adecuado" para el tipo de sociedad industrial y urbana que se generalizó progresivamente durante el presente siglo; no tuvieron, por lo tanto, necesidad de optar por la movilidad a lo largo de su curso de vida y, en gran medida, no lo hicieron. Fueron los nacidos en hábitats rurales los que desarrollaron una trayectoria migratoria activa, y los que tienen en la actualidad, además, la opción de retornar a sus lugares de origen; de ahí esos resultados tan diferenciados.

La duración del período sedentario que lleva el individuo viviendo en el mismo municipio se demuestra claramente relevante, independientemente de cuáles sean otros aspectos de su historia migratoria. Su efecto se mantiene en el análisis conjunto, de forma que sufren un fuerte descenso las probabilidades de migrar a medida que se incrementa el número de años que lleva el individuo residiendo en el mismo lugar. Conforme aumenta la duración de la residencia en un determinado municipio, el individuo va estableciendo vínculos con el entorno, que se fortalecen con la permanencia en el mismo, y las actividades cotidianas van delimitando un espacio de vida (Hägerstrand, 1970), de forma paralela al establecimiento de una serie de redes sociales; todo ello contribuye a «enraizar» al sujeto a un determinado territorio, dificultando la toma de una decisión que cambiará totalmente el espacio de vida, como es la de una migración.

Las probabilidades de migración respecto al tamaño demográfico del municipio de residencia previa confirman las mayores probabilidades de migración de aquellos que residen en áreas metropolitanas. Las grandes áreas urbanas ejercen un efecto neto de expulsión sobre la población de edad, independientemente de que sea en ellas en donde residen en su mayoría aquellos colectivos cuya historia migratoria también se traduce en una mayor propensión hacia la movilidad. El entorno metropolitano supone, por sí mismo, un medio poco atractivo para una población mayor que busca tranquilidad y un entorno agradable para el ocio y el descanso, y que percibe el medio urbano como un espacio inseguro y desapacible (Abellán, 1993; Cribier y Kych, 1993).

El número de migraciones previas presenta también un efecto dinamizador de la movilidad independientemente de las pautas geográficas (hábitat de nacimiento/hábitat de residencia 
actual) que haya dibujado la historia migratoria previa. Las probabilidades de migrar en la vejez aumentan a medida que aumenta el número de migraciones previas. El comportamiento migratorio en la vejez difiere de forma notoria entre aquellos que migraron alguna vez y los que no lo hicieron nunca. Entre los migrantes, las diferencias de patrón residencial tras los 55 años también son significativas entre aquellos que se han movido tan sólo en una ocasión y los muy móviles, pero no se ha de olvidar que tan sólo un 9\% de la población española mayor de 55 años ha realizado tres o más movimientos migratorios antes de esa edad.

No sólo la experiencia migratoria previa aporta una mayor propensión a la movilidad (o una menor resistencia a ella), sino que, tras toda una vida residiendo en el mismo entorno, las opciones residenciales en la vejez son muy reducidas y la probabilidad de embarcarse en un cambio residencial, por el que no se ha optado en edades más jóvenes, parece lejana.

Respecto a la tenencia de la vivienda, aun mostrando probabilidades diferenciadas de migración en la vejez, éstas son mucho menores al introducir la historia migratoria; en concreto, es importante controlar los efectos encubiertos de la duración del período sedentario, o años de residencia en el mismo municipio. El régimen de alquiler mantiene, pese a ello, unas probabilidades un $23 \%$ superiores de cambio residencial que la propiedad del inmueble, que, independientemente de la historia migratoria individual, frena la movilidad, actuando de «ancla» y fijando a la población al territorio en mayor medida que otras formas de tenencia.

\subsection{Análisis diferencial por género}

Se ha realizado el análisis por separado para ambos sexos con la pretensión de determinar si, aun a idénticos niveles de movilidad, los factores determinantes de la migración en la vejez varían para hombres y mujeres, o si alguno de ellos se traduce en probabilidades diferenciales por género. Como se puede apreciar en la tabla 2, la historia migratoria previa pesa de forma muy similar para hombres y mujeres a la hora de potenciar o restringir sus probabilidades de migración en la vejez. El tamaño demográfico, tanto de nacimiento como de residencia previa, así como el número de migraciones realizadas con anterioridad, juegan un papel muy similar en la movilidad tanto masculina como femenina.

Los años de residencia en el mismo municipio parecen fijar en mayor medida al territorio a las mujeres que a los varones. Mientras para los hombres mayores un período sedentario superior a quince años en el mismo municipio se traduce en unas probabilidades de migración 3 veces menores que aquellos que llevan entre diez y catorce años de residencia sedentaria, las muje- 
TABLA 2

Probabilidades relativas de migración en la vejez según historia migratoria previa, diferencias por género

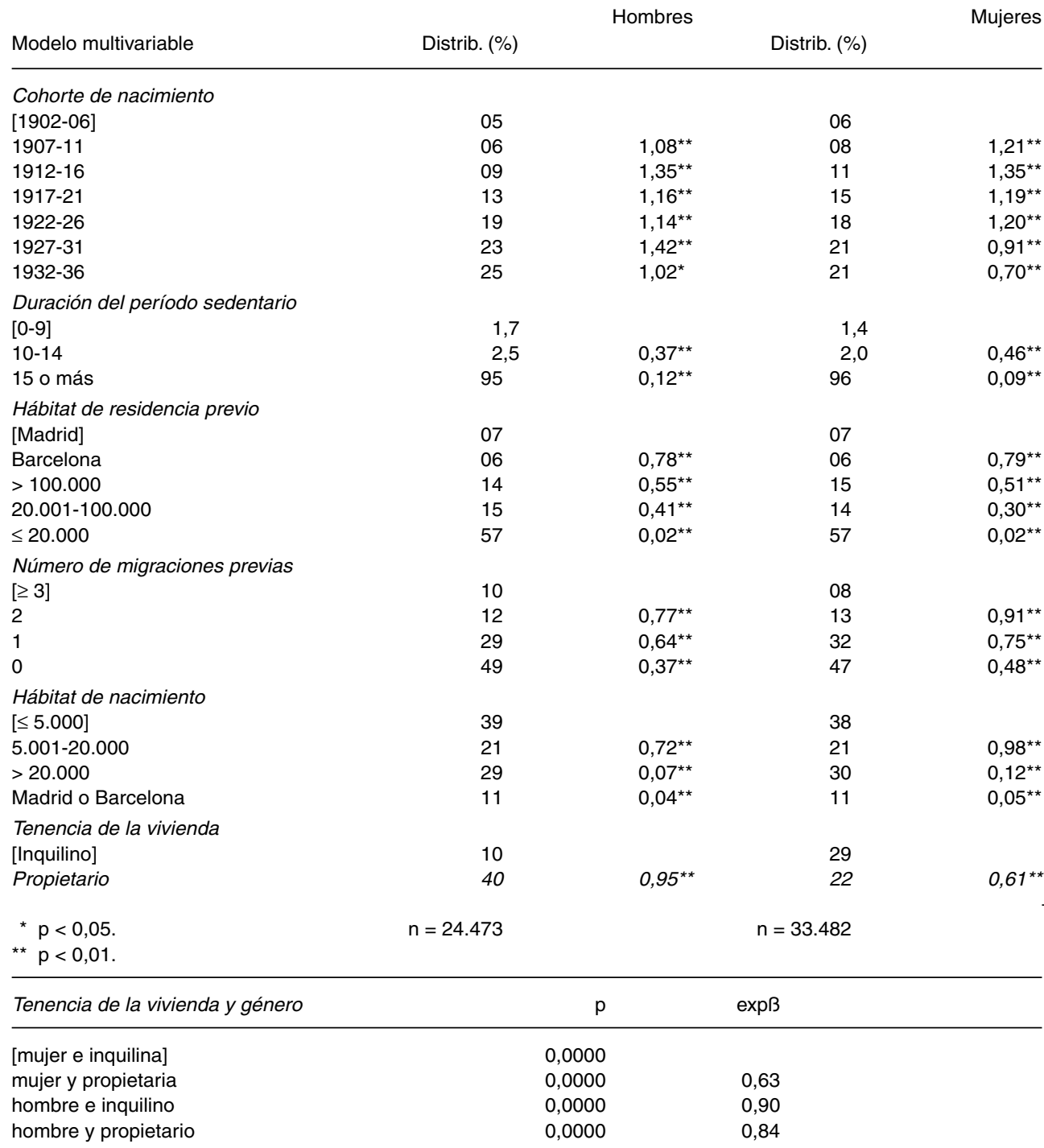

Modelo multavariable controlado por: cohorte de nacimiento, hábitat de nacimiento, número de migraciones previas, duración del período sedentario y previo hábitat de residencia.

Categorías de referencia entre corchetes.

FUENTE:

Elaboración propia a partir de los datos primarios de la ESD91. 
res de edad que llevan más de quince años residiendo en el mismo lugar presentan unas probabilidades de migración cinco veces menores que aquellas que llevan un período más reducido. No se ha de olvidar que la mayoría de las mujeres de estas generaciones o no se han incorporado jamás al mercado laboral o lo han hecho durante un período reducido de años, abandonándolo a edades jóvenes (un $40 \%$ de las que trabajaron alguna vez abandonaron la actividad productiva remunerada en el período de un año antes o después de contraer matrimonio). Esta diferente relación con la actividad remunerada ha significado una fuerte diferenciación por género de los papeles productivo y reproductivo. Como consecuencia, las mujeres de estas generaciones han desarrollado mayores vínculos con el espacio de vida inmediato, sintiéndose más ligadas espacial y socialmente al lugar de residencia (Abellán y Puga, 1999).

La cohorte de nacimiento presenta también efectos diferenciados sobre la migración en la vejez para hombres y mujeres. Las mujeres mayores muestran probabilidades superiores a las de los hombres en las primeras cohortes, frente a probabilidades un $30 \%$ inferiores a las de los varones en el caso de las más jóvenes. Ello parece indicar que son las mujeres las más vulnerables a las situaciones coyunturales, viéndose sus pautas migratorias mucho más alteradas por las mismas.

Finalmente, la que presenta claras diferencias por género a la hora de potenciar la migración en este período de la vida es la tenencia de la vivienda. Mientras para los varones no parece jugar un papel importante, presentando probabilidades de movilidad prácticamente idénticas para inquilinos y propietarios, las mujeres en régimen de alquiler tienen una probabilidad de migrar un $40 \%$ superior a la de las propietarias. Dicho efecto se ve confirmado al realizar el análisis (controlando por las mismas variables independientes) para la combinación de tenencia previa de la vivienda y sexo. El resultado confirma la significación de dichas diferencias, resultando que las probabilidades de migración de los hombres tan sólo varían en un $6 \%$ entre inquilinos y propietarios, mientras que en el caso de las mujeres el alquiler se traduce en una probabilidad superior en más de un $35 \%$ a la de las propietarias.

Nuevamente se ha de hacer constar una mayor vulnerabilidad femenina a la hora de mantener la estabilidad residencial. Como ya se ha comentado, la mayoría de las mujeres de edad no han desarrollado una trayectoria laboral remunerada (o lo han hecho durante un corto período de tiempo), por lo que llegadas a estas edades dependen económicamente de las pensiones de jubilación de sus cónyuges y, de forma creciente, de las pensiones de viudedad. Las pensiones de viudedad en España suponen aproximadamente la mitad de la pensión que recibía el titular (un $45 \%$ de la base reguladora). Es decir, la mayoría de estas mujeres, en el momento en que llega la viudedad ven reducido su nivel adquisitivo a menos de la mitad del que tienen los varones, lo que se puede traducir en mayores dificultades a la hora de mantener una residencia que exige el pago de una mensualidad. 


\section{CONCLUSIONES}

La vejez parece arrojar cierta invisibilidad sobre buena parte de los procesos que ocurren en la misma. La movilidad parece también ausente de ella, cuando no lo está en absoluto, quizás porque se ha asociado a menudo con la biografía laboral, olvidando una parte importante de la adoptada incluso durante la juventud y la edad adulta. De mantenerse los patrones de las generaciones de edades más avanzadas, casi uno de cada cinco individuos adoptará una estrategia residencial de movilidad durante su trayectoria a través de la vejez.

Cuando el envejecimiento es considerado como un proceso longitudinal, continuo a lo largo de la vida, la situación en cada momento de la biografía de un individuo es construida por la acumulación de la experiencia anterior y, a su vez, es la base de los eventos futuros. En las edades avanzadas la herencia biográfica es ya larga. Lo vivido, el curso de vida previo, aporta buena parte de las oportunidades, las restricciones y los recursos con los que afrontar la vejez.

Así, una trayectoria sedentaria dificulta enormemente el planteamiento de una estrategia de movilidad residencial en la vejez, cuando no se conoce otro espacio que el vivido. De la misma forma, cuando en la juventud no se ha optado por la movilidad más que en aquellos momentos en los que eventos de gran importancia (el matrimonio, el nacimiento de un hijo) se tradujeron en el comienzo de un nuevo recorrido biográfico, y por tanto impusieron el planteamiento —o la búsqueda - de un escenario para el mismo, llegada la vejez no se abandonará fácilmente ese escenario, salvo que el evento ocurrido se convierta en una ruptura biográfica de, al menos, idéntica fuerza que las de la juventud. Pero, ciertamente, en la vejez ocurren algunas de estas rupturas.

Por ello, a lo largo de este artículo se ha analizado la influencia de la biografía migratoria previa sobre las estrategias residenciales de movilidad en la vejez. La trayectoria migratoria previa resulta no sólo en la situación residencial del mayor al comenzar la vejez, sino también en su posición y las alternativas con las que cuenta ante la misma. Las decisiones previas, creadoras de continuidad a lo largo del curso de vida del individuo, se convierten en valiosos factores explicativos a la hora de entender la relación de los mayores con el territorio, es decir, el comportamiento residencial en la vejez.

Al comenzar la vejez, las herencias que le ha ido dejando al individuo el curso de vida previo son muchas; una de ellas es, en la mayoría de los casos, un largo período de residencia en el mismo municipio. Conforme aumenta la duración de la residencia en un lugar, el individuo va estableciendo vínculos con el entorno, que se fortalecen con la permanencia 
en el mismo; las actividades cotidianas van delimitando un espacio de vida, de forma paralela al establecimiento de una serie de redes sociales; todo ello contribuye a enraizar al sujeto a un determinado territorio, dificultando la toma de una decisión de movilidad. La duración del período sedentario se ha revelado fuertemente determinante de las decisiones residenciales durante la vejez. Si en los momentos biográficos en los que se abordó una estrategia residencial previa, en la mayoría de los casos, ésta supuso una ruptura con el espacio vivido durante veinte o veinticinco años, cuando esto ocurre en la vejez, normalmente se traduce en la ruptura con el entorno conocido y vivido durante cuarenta años, cuando no sesenta, o setenta, $u$ ochenta. En una sociedad en la que la movilidad hubiese estado más presente en la vida de las personas, y no tan sólo unida a unos pocos momentos únicos en sus biografías, segundos y posteriores movimientos hubiesen acortado los períodos de sedentarismo, llegando el individuo a la vejez con unas ligaduras menos fuertes con el espacio de residencia, así como con un mayor abanico de lugares vividos que, probablemente, le abrirían mayores opciones.

El entorno geográfico de nacimiento marcó la historia migratoria posterior de estos individuos, llegando a afectar incluso a la movilidad en la vejez, sobre la que muestra un claro efecto diferenciador. Los nacidos en áreas metropolitanas no tuvieron la misma necesidad de optar por la movilidad a lo largo de su curso de vida, y en gran medida no lo hicieron, presentando en la vejez unas menores probabilidades de abandonar un ámbito en el que ha transcurrido el curso de vida de la mayoría de ellos. Son, por el contrario, los nacidos en hábitats rurales los que desarrollaron una trayectoria migratoria activa, y los que cuentan en la actualidad con la opción de retornar a sus lugares de origen. La experiencia migratoria previa aporta una mayor propensión a la movilidad a edades avanzadas. Por el contrario, toda una vida en el mismo entorno geográfico y social reduce las opciones de optar en la vejez por una movilidad por la que no se decidieron a edades más jóvenes.

A ello se añade el efecto del entorno espacial en el que el individuo reside durante la vejez. Las grandes áreas urbanas ejercen un efecto de expulsión sobre la población de edad, independientemente de que sea en ellas en donde residen en su mayoría aquellos colectivos cuya historia migratoria también se traduce en una mayor propensión hacia la movilidad. El entorno metropolitano supone, por sí mismo, un medio poco atractivo para una población mayor que busca tranquilidad y un entorno agradable para el ocio y el descanso, y que percibe el medio urbano como un espacio inseguro y desapacible.

Finalmente, la menor tendencia a la movilidad en la vejez que presentan las generaciones nacidas entre 1907 y 1926 ha demostrado ser el resultado de la diferente historia migratoria vivida por estos individuos. De esta forma, los resultados han revelado que el descenso del riesgo de migración en la vejez que experimentan las generaciones nacidas en este pe- 
ríodo no es producto de un efecto generacional —por influencia del momento histórico-, sino que es efecto de las diferentes trayectorias migratorias vividas por las mismas.

Las trayectorias biográficas y, en concreto, migratorias previas son, pues, más determinantes de la migración en la vejez que las coyunturas puntuales. En el curso de vida avanzado, la migración es el resultado de las necesidades y oportunidades generadas por una ya extensa biografía. Ello induce a pensar que posibles cambios en las trayectorias migratorias de las generaciones más jóvenes se puedan traducir en un cambio sustantivo en los niveles de movilidad en la vejez, por encima de una situación coyuntural de un momento dado.

\section{BIBLIOGRAFÍA}

ABELLÁN, A. (1993): «La decisión de migrar en las personas de edad», Estudios Geográficos, 210, pp. 5-7.

ABELLÁN, A., y PUGA, D. (1999): «Movilidad residencial y género entre las personas de edad», Documents d'Analisi Geogràfica.

ALLISON, P. (1985): Event History Analysis: Regression for longitudinal Event Data, Sage Publications, Beverly Hills, $87 \mathrm{pp}$.

ANGEL, R. J.; ANGEL, J. L., y HIMES, C. L. (1992): «Minority group states, health transitions, and community living arrangements among the elderly", Research on Aging, 14, pp. 496-521.

BONVALET, C., y FRIBOURG, A. M. (1988): Stratégies résidentielles, INED, Congrès et Colloques 2, Paris, France, $459 \mathrm{pp}$.

CASTRO, T. (1992): «Delayed childbearing in contemporary Spain: trends and differentials», European Journal of Population, 8, pp. 217-246.

CLARK, R., y WOLF, D. (1992): «Proximity of children and elderly migration», en A. Rogers (ed.), Elderly Migration and Population Redistribution, Wiley, Sussex, pp. 77-96.

COURGEAU, D. (1980): Analyse Quantitative des Migrations Humaines, Masson, Paris, France, 225 pp.

- (1988): Methodes de mesure de la mobilite spatiale: migrations internes, mobilite temporaire, navettes, INED, Paris, France, $301 \mathrm{pp}$.

COURGEAU, D., y LELIEVRE, E. (1989): Analyse demographique des biographies, Editions de l'INED, Paris, France, $268 \mathrm{pp}$.

CRIBIER, F. (1975): «Retirement migration in France», en L. A. Kosinski y R. Mansell (eds.), People on move. Studies on internal migration, Methuen, London, pp. 361-373.

- (1980): «A European assessment of aged migration», Research on Ageing, 2, pp. 255-270.

- (1994): «La migration de retraite des Parisiens: l'apport des enquetes biographiques par cohortes», Espace, Populations, Sociétés, 1, pp. 75-83. 
CRIBIER, F., y KYCH, A. (1992): «La migration de retraite des Parisiens. Une analyse de la propension au départ», Population, 3, pp. 677-718.

- (1993): «A comparison of retirement migration from Paris and London», Enviroment and Planning A, 25, pp. 13991420.

ELCHARDUS, M. (1984): "Life cycle and life course. The scheduling and temporal integration of life», en S. Feld y R. Lesthaeghe (eds.), Population and societal outlook, Fondation Roi Baudouin, Brussels, Belgium, 320 pp.

FLYNN, C. B., et al. (1985): «The Redistribution of America's Older Population: Major National Migration Patterns for Three Census Decades, 1960-1980», The Gerontologist, 25, 3, pp. 292-296.

FOKKEMA, T. (1996): Residential Moving Behaviour of the Elderly. An explanatory Analysis for The Netherlands, The Tinbergen Institute Research Series 112, Thesis Publishers, Amsterdam, The Netherlands, $376 \mathrm{pp}$.

GARCÍA BARBANCHO, A. (1967): Las migraciones interiores en España. Estudio cuantitativo desde 1900, Instituto de Desarrollo Económico, Madrid, Spain, 209 pp.

GOLANT, S. M. (1982): «Individual differences underlaying the dwelling satisfaction of the elderly», Journal of Issues, 38 (3), pp. 121-133.

HÄGERSTRAND, T. (1970): «What about People in Regional Science», Papers of the Regional Science Association, 24 (1), pp. 7-21.

HEATON, T., y VAUGHN, R. A. (1995): «Modeling Family Dynamics with Event History Analysis», Journal of Marriage and the Family, 57, pp. 1078-1090.

LASLETT, P. (1991): A fresh map of life: The emergence of the third age, Weidenfeld \& Nichols, London.

LAW, C. M., y WARNES, A. M. (1982): «The destination decision in retirement migration», en A. M. Warnes (ed.), Geographical Perspectives on the elderly, cap. 3, pp. 53-81.

MANTON, K. G. (1988): «A longitudinal study of functional change and mortality in the United States», Journal of Gerontology, 43, pp. 153-161.

MEYER, J. W. (1986): «The self and the life course: Institutionalization and its effects», en A. B. Sorensen, F. E. Weinert y L. R. Sherrod (eds.), Human development and the life course: Multidisciplinary perspectives, Hillsdale, Erlbaum, pp. 199-245.

MEYER. J. W., y SPEARE, A. (1985): «Distinctively elderly mobility: types and determinants», Economic Geography, 61,1, pp. $79-88$.

MULDER, C. (1993): Migration dynamics: a life course approach, PDOD Publications Series A: Doctoral Dissertations, Thesis Publishers, Amsterdam, Netherlands, $251 \mathrm{pp}$.

NADAL, J. (1984): La población española (siglos xVI a xx), Ariel, Barcelona, 264 pp.

PUGA, D. (2000): «Pautas migratorias de los mayores en España: intensidad y calendario», Revista Internacional de Sociología, 27, pp. 23-40.

PUGA, D., y ABELLÁN, A. (1998): «El primer movimiento migratorio en la biografía de los españoles», Estudios Geográficos, LIX (233), pp. 689-709.

PUJADAS, I.; GARCÍA, A., y PUGA, D. (1995) «Migraciones interiores en España: tendencias recientes y perspectivas de futuro (1971-2000)», Revista de Geografía, XXIX (3), pp. 5-150.

REHER, D. (1996): La familia en España. Pasado y presente, Alianza Universidad, Madrid, Spain, 470 pp. 
RINDFUSS, R. R.; SWICEGOOD, C. G., y ROSENFELD, R. A. (1987): «Disorder in the life course: how common and does it matter? », American Sociological Review, 52 (6), pp. 785-801.

ROGERS, A., y CASTRO, L. J. (1984): «Model migration schedules», en A. Rogers (ed.), Migration, urbanization and spatial population dynamics, Westview Press, Boulder, pp. 41-69.

ROSEMAN, C. (1971): «Migration as a spatial and temporal process», Annals of the Association Geography, 61 (3), pp. 589-598.

SEROW, W. J. (1987): «Determinants of interstate migration: differences between elderly and nonelderly movers», Journal of Gerontology, 42, 1, pp. 95-100.

SPEARE, A., y MEYER, J. (1988): «Types of Elderly Residential Mobility and Their Determinants», Journal of Gerontology: Social Sciences, 43 (3), pp. 574-581.

STOKOLS, D., y SHUMAKER, S. A. (1982): «The Psychological context of residential mobility and well-being», Journal of Social Issues, 38, 3, pp. 149-171.

TEACHMAN, J. (1982): “Methodological Issues in the Analysis of Family Formation and Dissolution», Journal of Marriage and the Family, 44, pp. 1037-1053.

VARADY, D. P. (1980): «Housing problems and mobility plans among the elderly», Journal of the American Planning Association, 46, 3, pp. 301-314.

VERGOOSSEN, D., y WARNES, A. (1989): «Migration of the elderly», en J. Stillwell y H. Scholten (eds.), Contemporary research in population geography: a comparison of the United Kingdom and the Netherlands, Kluwer Academic, Boston, pp. 129-143.

WAGNER, M. (1990): «Education and migration», en K. U. Mayer y N. B. Tuma, Event history analysis in life course research, Life Course Studies, University of Wisconsin Press, Madison, Wisconsin, 297 pp.

WARNES, A. (1993): «Migration and life course», en T. Champion y T. Fielding (eds.), Migration patterns and processes. Volume 1: research progress and prospects, Belhaven Press, New York, New York/London, England, 256 pp.

WILLEKENS, F. (1991): «Understanding the interdependence between parallel careers», en J. J. Siegers, J. de JongGierveld y E. van Imhoff (eds.), Female labour market behaviour and fertility: A rational-choice approach, SpringerVerlag, Berlin, Germany, $301 \mathrm{pp}$.

WISEMAN, R. F. (1980): «Why Older People Move», Research on Aging, 2 (2), pp. 141-154.

WOROBEY, J. L., y ANGEL, R. J. (1990): «Functional capacity and living arrangements of unmarried elderly persons», Economic Geography, 55, pp. 324-337. 


\section{ABSTRACT}

In the present-day ageing societies, residential conduct of the elderly is acquiring increasing importance. Explored in this article are the causal relationships between prior migratory biography and the residential guidelines adopted after reaching the age of 55, using Event History Analysis. The results show that the place of birth marks the subsequent migratory trajectory of the individuals involved, and can even come to effect residential conduct in old age. The existence of previous migrations, residence habitat, as well as the duration of the sedentary period also exert a considerable influence on the decision for mobility in old age. Lastly, it is shown that reduction in mobility among the most recent generations of elderly people is an effect resulting from their previous migratory biography. Migratory trajectories protagonized by individuals during their young and adult life show themselves to be more decisive in respect of residential conduct in old age than circumstances relating to the moment. For this reason, possible changes in the migratory biographies of the younger generations could also turn into changes in levels of mobility in old age, irrespective of the circumstances of the moment. In this respect we can observe gender differences, in which women are more vulnerable than men when faced with historical circumstances.

Key words: Ageing, Residential Strategies, Mobility, Life Quality Among the Elderly, Life Course, Migration History, Event History Analysis. 